\title{
NUEVA VISIÓN DE LAS RELACIONES FAMILIARES: REFLEXIONES A PARTIR DE LA ACTUAL LEGISLACIÓN ARGENTINA
}

María Isabel Sokolich Alva ${ }^{1}$

\section{RESUMEN}

Las nuevas tendencias del derecho de familia son el resultado, en muchos de los casos, de cambios sociales, estructurales y avance de la ciencia, los que al influir en los tradicionales conceptos de familia y relaciones interpersonales, merecen un enfoque distinto por el legislador a fin que la norma resulte conforme la realidad. Por el presente artículo se analizarán algunos aspectos de la actual legislación civil argentina en contraposición con la vigente legislación nacional.

\section{ABSTRACT}

New trends in family law are the result, in many cases, of social and structural changes and the advancement of science. These influence the traditional concepts of family and interpersonal relationships and, hence, deserve to be properly addressed by lawmakers so that the new laws conform to reality. This paper analyzes the current Argentine civil law as opposed to the current national legislation.

\section{PALABRAS CLAVE:}

Derecho al nombre. Filiación. Matrimonio Civil. Divorcio

\section{KEYWORDS}

Right to a Name. Filiation. Civil Marriage. Divorce

El 01 de octubre de 2014, el Congreso Argentino promulgó la Ley N. ${ }^{\circ} 26.994$, por la cual se aprobó un nuevo Código $\mathrm{Ci}$ vil y Comercial de la Nación, el cual conforme a lo dispuesto por Ley $\mathrm{N}^{\circ}{ }^{\circ} 27.077$, entró en vigencia el $1^{\circ}$ de agosto de 2015. Los cambios que plantea este nuevo ordenamiento legal, específicamente en lo referente a la regulación de la familia y las relaciones derivadas de ella, merecen especial atención, pues se reconoce la realidad imperante y los cambios sociales que directamente influyen sobre los tradicionales conceptos de familia, y, por ende, en las relaciones interpersonales y familiares. Ello nos lleva a analizar si las nor- mas nacionales en materia de nombre, filiación, matrimonio, divorcio, entre otras, resultan acordes a dichos cambios. A continuación procederemos a reflexionar al respecto:

\section{a) El derecho al nombre:}

El artículo $19 .^{\circ}$ del Código Civil Peruano establece que toda

\footnotetext{
Fiscal Adjunto Supremo. Doctora en Derecho. Magíster en Derecho Civil con mención en Derecho de Familia. Docente de la Maestría de Derecho Civil con mención en Derecho de Familia de la Universidad Femenina del Sagrado Corazón -UNIFE-, así como de la Maestría de Derecho de Persona, Matrimonio y Familia de la Universidad Católica Santo Toribio de Mogrovejo. Docente de la Academia de la Magistratura en los cursos de Derecho de Familia. Autora del libro "Violencia Familiar" y de diversos artículos jurídicos; expositora en diversos eventos académicos relacionados con el Derecho de Familia.
} 
persona tiene el derecho y el deber de llevar un nombre, lo que incluye los apellidos. El nombre, como sabemos, es un atributo esencial del derecho a la identidad, pues a la vez que nos individualiza y distingue de los demás, nos vincula con una estirpe, $y$, por ende con una familia.

El Tribunal Constitucional, por la sentencia recaída en el Exp. N. ${ }^{\circ}$ 2273-2005-PHC/TC LIMA (Karen Mañuca Quiroz Cabanillas), recalcó que “(...) el nombre es la designación con la cual se individualiza al sujeto y que le permite distinguirse de los demás. El nombre tiene dos componentes: el prenombre y los apellidos. El nombre es el elemento característico individual del sujeto, libre de toda vinculación preestablecida. Se refiere al nombre de pila, el cual es libre y es elegido por los padres o por el que hace la inscripción en el registro civil. La elección de un segundo o más nombres es facultativa. El nombre recoge datos históricos de la persona que la singularizan de los demás y provee la información base para la emisión del DNI. Es obligatorio tenerlo y usarlo; es inmutable, salvo casos especiales; no es comercial, puesto que es personalísimo, aun cuando se transmita por procreación; es imprescriptible, aunque se deje de usar, se haya empleado uno más o menos erróneo o se utilice un conocido seudónimo. Asimismo, permite la identificación, individualización y la pertenencia de una persona a una familia. Mediante el nombre se hace posible el ejercicio de derechos tales como la ciudadanía, la educación, la seguridad social, el trabajo y la obtención de una partida de nacimiento, entre otros".

En cuanto a los apellidos del hijo, como componentes del nombre, el artículo $20 .^{\circ}$ del Código Civil establece que al hijo le corresponde el primer apellido del padre y el primero de la madre.

El orden de los apellidos es establecido por el legislador con el afán de organizar la identificación de las personas; sin embargo, este hecho de preferir en primer lugar el apellido del padre ha sido cuestionado por considerarse un rezago imperante del machismo o una extensión del poder absoluto del pater familias, en desmedro de igual derecho o interés de la madre.

Así, la Corte Constitucional de Colombia por sentencia No. C-152/94, de fecha 24 de marzo de 1994, con motivo de la demanda de inconstitucionalidad $^{2}$ planteada contra la Ley 54 de 1989, por la cual se reformó el artículo 53. ${ }^{\circ}$ del decreto 1260 de $1970^{3}$, esbozó como argumentos para declarar EXEQUIBLE la norma en referencia los siguientes:

“(...) Si el nombre, es decir, el nombre de pila y el apellido, "es el signo distintivo y revelador de la personalidad", y es a la vez un "procedimiento de identificación", interesa a la sociedad la existencia de un orden en la manera de fijarlo. Dicho en otras palabras: como todos los hechos y actos relativos al estado civil están regidos por normas de orden público, inderogables y forzosas, no podría dejarse la determinación del nombre, librada al capricho de los particulares.

En consecuencia, el nombre es un elemento del estado civil, regulado por la ley, por mandato expreso del último inciso del artículo 42 de la Constitución : "La ley determinará lo relativo al estado civil de las personas y los consiguientes derechos y deberes".

En resumen: la manera como se determine el nombre, obedece a una finalidad social y su regulación corresponde a la ley.

c) El orden de los apellidos en la inscripción del nacimiento. Si en la inscripción del nacimiento, se inscriben dos apellidos, uno de ellos debe ser el primero, y el otro el segundo. La ley ha determinado un or-

Fundamento jurídico $13 .^{\circ}$

El demandante Rafael Soto Beltrán alegó que el artículo 1o. de la ley 54 de 1989, que modificó el 53 del decreto 1260 de 1970, era contrario a la Constitución al establecer que en el registro de nacimientos se inscribirán como apellidos del inscrito, el primero del padre, seguido del primero de la madre. Dicho orden, a decir del actor constituía discriminación contra la madre, al violar los artículos 13, 42, 43 y 44 de la Constitución Colombiana que consagran la igualdad de las personas ante la ley, y la igualdad entre el hombre y la mujer.

3 ARTICULO 10.- El artículo 53 del Decreto 1260 de 1970, quedará así: “ Artículo 53: En el registro de nacimiento se inscribirán como apellidos del inscrito, el primero del padre, seguido del primero de la madre, si fuere hijo legítimo o extramatrimonial reconocido o con paternidad judicialmente declarada; en caso contrario, se le asignarán los apellidos de la madre. 
den, es decir, ha reglamentado el nombre, elemento del estado civil.

¿Podría dejarse esta materia al arbitrio de los particulares, para que ellos, y no la ley, establecieran el orden? Evidentemente, la ley podría establecerlo así. Pero ello crearía el desorden y haría difícil la identificación de las personas: en una familia habría, por ejemplo, hermanos carnales que llevarían primero el apellido paterno, y otros el materno.

Pero, por el hecho de definir los padres, a veces en medio de disputas, el orden de los apellidos, ¿se avanzaría en el camino de la igualdad? Evidentemente, no, y ello por una razón elemental: el orden de los apellidos del hijo, nada significa en relación con sus derechos, ni con los de los padres.

Es claro, en consecuencia, que el orden de los apellidos en la inscripción en el registro de nacimiento, nada tiene que ver con la igualdad de derechos y obligaciones. Tiene que existir un orden, $y$ la ley lo ha determinado (énfasis agregado).

En minoría, los magistrados Eduardo Cifuentes Muñoz, Carlos Gaviria Díaz y Alejandro Martinez Caballero, por salvamento de voto, expresaron lo siguiente:

"La circunstancia de que la ley (en sentido material) dispon- ga que al inscribirse un hijo "legítimo o extramatrimonial reconocido o con paternidad judicialmente declarada" se registre en primer lugar el apellido del padre, no es inocua sino marcadamente significativa: es el trasunto de una milenaria tradición patriarcal que relega a la mujer a un plano secundario, porque la prevalencia del hombre se asume como un hecho indiscutido. Argüir que la ley se ha limitado a recoger un uso social muy extendido, en el espacio y en el tiempo, equivale a soslayar el problema, pues de lo que se trata es de saber qué razones avalan la existencia de tal uso y si ellas están en armonía con los propósitos consignados en la norma suprema del ordenamiento. En el caso sub-judice, no hay duda de que no se ha dado carta en blanco al legislador para que disponga lo que a bien tenga, con total desentendimiento de un principio como el de la igualdad, informante de toda la Carta del $91 \mathrm{y}$, particularmente, de las relaciones familiares que, bajo esta perspectiva, sufrieron un vuelco radical con respecto a la Constitución anterior. Es claro, para quienes suscribimos este salvamento, que la norma acusada padece de inconstitucionalidad sobreviniente".

\section{(...)}

"El argumento esgrimido en beneficio de la constitucionalidad de la norma atacada, en el sentido de que con ella se pretende implantar cierto orden en la identificación de los miembros de una familia, es igualmente inane, puesto que dicha uniformidad se lograría también si se diera prelación al apellido de la madre o, lo que parece más sensato, si el orden de los apellidos se estableciera por acuerdo mutuo del hombre y la mujer, lo que sí resultaría armónico con la igualdad de derechos que la Carta del 91 predica de ambos". (énfasis agregado).

Como es de advertir, si bien por mayoría se resolvió a favor de la constitucionalidad de la norma, lo cierto es que el hecho de preferir el apellido del padre sobre el de la madre, implica, de alguna forma, el empoderamiento que le confiere la sociedad al varón sobre los derechos de los hijos ${ }^{4}$, en desmedro de los derechos de la mujer y madre, respectivamente; esta situación fáctica ha sido superada por la legislación argentina por el actual artículo $64 .^{\circ}$ al establecer taxativamente lo siguiente:

>> Apellido de los hijos. El hijo matrimonial lleva el primer apellido de alguno de los cónyuges; en caso de no haber acuerdo, se determina por sorteo realizado en el Registro del Estado Civil y Capacidad de las Personas. A pedido de los padres, o del interesado con edad y madurez suficiente, se puede agregar el apellido del otro.

Todos los hijos de un mismo matrimonio deben llevar el

4 De allí que lo correcto es aludir a "responsabilidad parental" en vez de "patria potestad", toda vez que éste último término alude exclusivamente al poder o autoridad del padre sobre los hijos. Por el contrario, el término responsabilidad parental involucra a "ambos padres" en la crianza y desarrollo integral de sus hijos, lo cual resulta acorde a la Convención sobre los Derechos del Niño.. 
apellido y la integración compuesta que se haya decidido para el primero de los hijos.

El hijo extramatrimonial con un solo vínculo filial lleva el apellido de ese progenitor. Si la filiación de ambos padres se determina simultáneamente, se aplica el primer párrafo de este artículo. Si la segunda filiación se determina después, los padres acuerdan el orden; a falta de acuerdo, el juez dispone el orden de los apellidos, según el interés superior del niño"

La norma así plasmada, deja en potestad de los padres decidir lo siguiente: a) Si el hijo llevará anexo a su prenombre solo un apellido. De ser así elegirán libre y voluntariamente entre el apellido del padre y el de la madre; y, b) Si el hijo llevará el apellido de ambos padres. En cuyo caso, igualmente acordarán el orden en el que se consignarán dichos apellidos. Solo en caso de falta de acuerdo, el hecho será resuelto al azar en presencia del funcionario del Registro de Estado Civil y Capacidad de las Personas.

Si bien para algunos, la solución planteada por la norma puede resultar novedosa, lo cierto y verdadero es que el legislador argentino pretende equiparar los derechos de los padres a la hora de determinar el nombre de los hijos, lo cual resulta coherente con los derechos fundamentales a la igualdad ante la ley y no discriminación.

En cuanto al derecho al nombre, otro aspecto importante de resaltar es el referente a la identificación de la "persona casada"; no exclusivamente de la mujer casada, sino, del hombre o mujer unido a otra persona en matrimonio. En la experiencia nacional, el artículo $24 .^{\circ}$ del Código Civil establece que "La mujer tiene derecho a llevar el apellido del marido agregado al suyo y a conservarlo mientras no contraiga nuevo matrimonio. Cesa tal derecho en caso de divorcio o nulidad de matrimonio"; vale decir, que solo la mujer tiene expedito el derecho de llevar anexo a su nombre de soltera la preposición "de" seguido del apellido paterno de su cónyuge.

Con el afán de resolver el problema, el vigente Código Civil y Comercial de la Nación de Argentina por el articulo $67 .^{\circ}$ prevé lo siguiente:

>>> "Cualquiera de los cónyuges puede optar por usar el apellido del otro, con la preposición "de" o sin ella.

La persona divorciada o cuyo matrimonio ha sido declarado nulo no puede usar el apellido del otro cónyuge, excepto que, por motivos razonables, el juez la autorice a conservarlo.

El cónyuge viudo puede seguir usando el apellido del otro cónyuge mientras no contraiga nuevas nupcias, ni constituya unión convivencial".

La norma así planteada, equipara los derechos de hombres y mujeres, de tal forma que el varón casado podrá, si así lo desea, identificarse con su prenombre seguido del apellido paterno o materno, al cual se le agregará la preposición "de" y el apellido de su cónyuge; de esta forma podríamos conocer, por ejemplo, de un varón que se identifica como Luis Céspedes de Martínez, lo cual denota que este señor está unido por el vínculo del matrimonio con la señora Martínez. La pregunta que surge es la siguiente: ien el Perú estamos preparados para un cambio normativo como el descrito?.

\section{b) Filiación:}

La filiación entendida como vínculo jurídico que une a padres con hijos y que tradicionalmente se ha entendido que tiene un origen por naturaleza (filiación matrimonial y extramatrimonial) y por adopción, como así regula la legislación nacional, cede paso a los cambios propiciados por el avance de la ciencia, de tal forma que el actual Código Civil y Comercial Argentino incluye a las técnicas de reproducción humana asistida (inseminación artificial, fecundación in vitro), como un nuevo origen de filiación.

Como sabemos, el deseo de una persona de ser padre o madre ya no se supedita necesariamente a la sexualidad entre un varón y una mujer, sino, que la sola "voluntad procreacional" es el punto de partida de la reproducción sin sexo. En efecto, en la actualidad, por la maternidad subrogada, por ejemplo, una mujer puede ser madre de un hijo sin necesidad de llevar adelante la gestación, pues el proceso de crecimiento y desarrollo del embrión se desarrollará en el útero de otra mujer.

Las técnicas de reproducción humana asistida han permitido, además, que parejas del 
mismo sexo, hombre y mujeres estériles o de edad avanzada puedan convertir su deseo de ser padres y madres en una realidad, acudiendo en muchos casos a los llamados bancos de "donantes" de espermatozoides y óvulos. Si bien el tema es controversial pues desnaturaliza el origen natural de las personas, lo cierto es que es un hecho que la legislación no puede desconocer.

Esta ha sido la razón por la cual el artículo $558 .^{\circ}$ del Código Civil y Comercial Argentina está redactado en los términos siguientes:

>>> “La filiación puede tener lugar por naturaleza, mediante técnicas de reproducción humana asistida, o por adopción.

La filiación por adopción plena, por naturaleza o por técnicas de reproducción humana asistida, matrimonial y extramatrimonial, surten los mismos efectos, conforme a las disposiciones de este Código.

\section{Ninguna persona puede tener} más de dos vínculos filiales, cualquiera sea la naturaleza de la filiación.

El legislador argentino ${ }^{5}$, asimismo, ha previsto determinadas reglas generales relativas a la filiación por técnicas de reproducción humana asistida como las siguientes: a) el consentimiento en las técnicas de reproducción humana asistida; b) la forma y requisitos del consentimiento; c) la voluntad procreacional; d) el derecho a la información de las personas nacidas por técnicas de reproducción asistida; y, e) el contenido de la información.

En el Perú, el artículo $7 .^{\circ}$ de la Ley N. ${ }^{\circ} 26842$, Ley General de Salud, establece que "Toda persona tiene derecho a recurrir al tratamiento de su infertilidad, así como a procrear mediante el uso de técnicas de reproducción asistida, siempre que la condición de madre genética y de madre gestante recaiga sobre la misma persona. Para la aplicación de técnicas de reproducción asistida, se requiere del consentimiento previo y por escrito de los padres biológicos. Está prohibida la fecundación de óvulos humanos con fines distintos a la procreación, así como la clonación de seres humanos". (énfasis agregado)

\section{c) Matrimonio:}

El Código Civil Peruano ${ }^{6}$ establece que los cónyuges por el matrimonio tienen deberes y derechos recíprocos, como son, fidelidad, asistencia y cohabitación, de tal forma que

5 ARTICULO 560.- Consentimiento en las técnicas de reproducción humana asistida. El centro de salud interviniente debe recabar el consentimiento previo, informado y libre de las personas que se someten al uso de las técnicas de reproducción humana asistida. Este consentimiento debe renovarse cada vez que se procede a la utilización de gametos o embriones.

ARTICULO 561.- Forma y requisitos del consentimiento. La instrumentación de dicho consentimiento debe contener los requisitos previstos en las disposiciones especiales, para su posterior protocolización ante escribano público o certificación ante la autoridad sanitaria correspondiente a la jurisdicción. El consentimiento es libremente revocable mientras no se haya producido la concepción en la persona o la implantación del embrión.

ARTICULO 562.- Voluntad procreacional. Los nacidos por las técnicas de reproducción humana asistida son hijos de quien dio a luz y del hombre o de la mujer que también ha prestado su consentimiento previo, informado y libre en los términos de los artículos 560 y 561, debidamente inscripto en el Registro del Estado Civil y Capacidad de las Personas, con independencia de quién haya aportado los gametos.

ARTICULO 563.- Derecho a la información de las personas nacidas por técnicas de reproducción asistida. La información relativa a que la persona ha nacido por el uso de técnicas de reproducción humana asistida con gametos de un tercero debe constar en el correspondiente legajo base para la inscripción del nacimiento.

ARTICULO 564.- Contenido de la información. A petición de las personas nacidas a través de las técnicas de reproducción humana asistida, puede:

a) obtenerse del centro de salud interviniente información relativa a datos médicos del donante, cuando es relevante para la salud;

b) revelarse la identidad del donante, por razones debidamente fundadas, evaluadas por la autoridad judicial por el procedimiento más breve que prevea la ley local.

Obligaciones comunes de los cónyuges

Artículo 287. - Los cónyuges se obligan mutuamente por el hecho del matrimonio a alimentar y educar a sus hijos.

Deber de fidelidad y asistencia

Artículo 288. - Los cónyuges se deben recíprocamente fidelidad y asistencia.

Deber de cohabitación

Artículo 289. - Es deber de ambos cónyuges hacer vida común en el domicilio conyugal. El juez puede suspender este deber cuando su cumplimiento ponga en grave peligro la vida, la salud o el honor de cualquiera de los cónyuges o la actividad económica de la que depende el sostenimiento de la familia. 
el incumplimiento de alguno de estos deberes puede dar lugar a la materialización de alguna de las causales de separación de cuerpos y divorcio que regula el artículo $333 .^{\circ}$ del mismo ordenamiento legal.

Al respecto, el actual ordenamiento legal argentino regula por el articulo $431 .^{\circ}$ que los esposos se comprometen a desarrollar un proyecto de vida en común basado en la cooperación, la convivencia y el deber moral de fidelidad.

La fidelidad, entonces, deja de ser un deber legal o jurídico para convertirse únicamente en una obligación moral; así planteada la norma, se entiende que los cónyuges tienen el deber ético de mantener solo relaciones sexuales entre ellos; sin embargo, el incumplimiento de dicho deber moral no da lugar a una sanción, por lo que el adulterio ya no es causal de divorcio.

\section{d) Divorcio:}

En cuanto a la disolución del vínculo matrimonial, desaparecen las trabas u obstáculos que hasta antes de la vigencia de la nueva norma implicaba que un cónyuge dentro de un proceso judicial demuestre que el otro (o ambos) había vulnerado alguno de los deberes nacidos del matrimonio; en otras palabras, la petición de divorcio no requiere se invoque causal alguna y prospera a solo pedido de uno o ambos cónyuges. De allí que se le haya denominado "Divorcio Express".

Con ello el legislador pretende equilibrar el inicio y el fin de la relación matrimonial, pues si aquella se concretó por decisión y complacencia de ambos cónyuges, no existe razón alguna para que en caso que el matrimonio no cumpla sus fines, uno de los cónyuges o ambos, mantengan el vínculo contra su voluntad.

La novedad del cambio legislativo es la inclusión de una "compensación económica"7 en caso que el divorcio origine a uno de los cónyuges un desequilibrio manifiesto que suponga un empeoramiento de su situación. Dicha compensación puede consistir en una prestación única, en una renta por tiempo determinado o, excepcionalmente, por plazo indeterminado y puede pagarse con dinero, con el usufructo de determinados bienes o de cualquier otro modo que acuerden las partes o decida el juez. La acción para reclamar la compensación económica caduca a los seis meses de haberse dictado la sentencia de divorcio.

Es importante resaltar, que para la fijación judicial de la compensación económica se han establecido determinados indicadores que deben ser evaluados por el Juez, como son los siguientes:

a) El estado patrimonial de cada uno de los cónyuges al inicio y a la finalización de la vida matrimonial;

b) La dedicación que cada cónyuge brindó a la familia y a la crianza y educación de los hijos durante la convivencia y la que debe prestar con posterioridad al divorcio;

c) La edad y el estado de salud de los cónyuges y de los hijos;

d) La capacitación laboral y la posibilidad de acceder a un

7 La compensación económica está recogida también en el artículo artículo $97 .^{\circ}$ del Código Civil Español:

El cónyuge al que la separación o el divorcio produzca un desequilibrio económico en relación con la posición del otro, que implique un empeoramiento en su situación anterior en el matrimonio, tendrá derecho a una compensación que podrá consistir en una pensión temporal o por tiempo indefinido, o en una prestación única, según se determine en el convenio regulador o en la sentencia.

A falta de acuerdo de los cónyuges, el Juez, en sentencia, determinará su importe teniendo en cuenta las siguientes circunstancias:

$1 .^{a}$ Los acuerdos a que hubieran llegado los cónyuges.

2. ${ }^{2}$ La edad y el estado de salud.

3. ${ }^{a}$ La cualificación profesional y las probabilidades de acceso a un empleo.

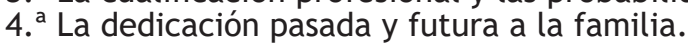

5. ${ }^{\text {a }}$ La colaboración con su trabajo en las actividades mercantiles, industriales o profesionales del otro cónyuge.

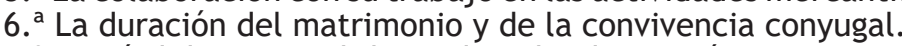

7. ${ }^{a}$ La pérdida eventual de un derecho de pensión.

8. ${ }^{\text {a }}$ El caudal y los medios económicos y las necesidades de uno y otro cónyuge.

9. ${ }^{a}$ Cualquier otra circunstancia relevante.

En la resolución judicial o en el convenio regulador formalizado ante el Secretario judicial o el Notario se fijarán la periodicidad, la forma de pago, las bases para actualizar la pensión, la duración o el momento de cese y las garantías para su efectividad. 
empleo del. cónyuge que solicita la compensación económica;

e) La colaboración prestada a las actividades mercantiles, industriales 0 profesionales del otro cónyuge; f) La atribución de la vivienda familiar, y si recae sobre un bien ganancial, un bien propio, o un inmueble arrendado. En este último caso, quién abona el canon locativo.

\section{BIBLIOGRAFÍA}

Código Civil y Comercial de la Nación -Argentina

Código Civil del Perú 
